\title{
Low Beta-Adrenergic Sweat Responses in Cystic Fibrosis and Cystic Fibrosis Transmembrane Conductance Regulator-Related Metabolic Syndrome Children
}

\author{
Danieli Barino Salinas, MD, MS,' Lucia Kang, MD, Colleen Azen, MS, and Paul Quinton, PhD,4
}

$\beta$-adrenergically stimulated sweat secretion depends on the function of the cystic fibrosis transmembrane conductance regulator (CFTR) and discriminates between cystic fibrosis (CF) patients and healthy controls. Therefore, we sought to determine the feasibility, safety, and efficacy of assaying $\beta$-adrenergic sweating in children identified by CF newborn screening to help determine prognoses for individuals with CFTR-related metabolic syndrome (CRMS). Preschool age children with a positive newborn screening test for CF participated in this cross-sectional study. Sweat rates were measured by evaporimetery (cyberDERM, inc.) as transepidermal water losses $\left(\mathrm{g} \mathrm{H}_{2} \mathrm{O} / \mathrm{m}^{2} / \mathrm{h}\right)$ before and after selectively stimulating sweat glands either cholinergically or $\beta$ adrenergically. Net peak sweat responses assayed as evaporation rates were compared between CF and CRMS cohorts. After a pilot test in adults, children between 4 and 6 years of age were evaluated $(\mathrm{CF}, n=16$; CRMS, $n=10$ ). The test protocol was well tolerated; electrocardiograms and vital signs were within normal range for all subjects. The mean evaporative sweat rates in both groups in response to cholinergic stimulation were similar $(\mathrm{CF}$, 60.3 \pm 23.8 ; CRMS, $57.7 \pm 13.9 ; p=0.72$ ) as well as to $\beta$-adrenergic stimulation (CF, $1.1 \pm 1.7$; CRMS, $2.0 \pm 2.0$; $p=0.14)$. The $\beta$-adrenergic sweat test is safe and well tolerated by young children. However, the $\beta$-adrenergic sweat secretion rates as measured by evaporimetery did not discriminate between CF and CRMS cohorts.

Keywords: cystic fibrosis, CRMS, sweat rate, beta-adrenergic stimulation, sweat chloride test

\section{Introduction}

$\mathrm{H}$ UMAN SWEAT GLANDS secrete fluid via cholinergically and adrenergically innervated pathways. The $\beta$-adrenergic secretory pathway is dependent on the cystic fibrosis transmembrane conductance regulator (CFTR) protein chloride channel. ${ }^{1}$ Therefore, subjects with cystic fibrosis $(\mathrm{CF})$ do not secrete in response to purely adrenergic agonists, but do secrete normally in response to cholinergic agonists. ${ }^{2,3}$ This pathophysiology provides an indication of the level of CFTR protein function for diagnosis, prognosis, and potential therapeutic responses. $^{4-6}$

Recent in vivo assays of $\beta$-adrenergic sweat secretion discriminated among subjects with variable CFTR function, CF, CFTR gene carriers, and noncarrier healthy controls. ${ }^{4,5}$ These assays may help to diagnose and determine prognoses for individuals carrying CFTR mutations of unknown clinical significance as seen in CFTR-related metabolic syndrome (CRMS), also known as newborn " $\mathrm{CF}$ screening positive, inconclusive diagnosis". 7,8

The aim herein was to determine the feasibility, safety, and efficacy of measuring $\beta$-adrenergic sweating by evaporimetery in children identified by newborn screening in California.

\section{Methods \\ Population}

Adults and children were recruited for a cross-sectional study. Healthy adult volunteers were tested to compare $\beta$-adrenergic responses with and without precholinergic

\footnotetext{
${ }^{1}$ Department of Pediatrics-Pediatric Pulmonology, Children's Hospital Los Angeles, Keck School of Medicine-University of Southern California (USC), Los Angeles, California.

${ }^{2}$ Southern California Clinical and Translational Science Institute, Children's Hospital Los Angeles, University of Southern California, Los Angeles, California.

${ }^{3}$ Department of Pediatrics, Rady's Children's Hospital, University of California, San Diego (UCSD), La Jolla, California.

${ }^{4}$ Department of Biomedical Sciences, UC Riverside (UCR), Riverside, California.

(C) Danieli Barino Salinas et al. 2017; Published by Mary Ann Liebert, Inc. This Open Access article is distributed under the terms of the Creative Commons Attribution Noncommercial License (http://creativecommons.org/licenses/by-nc/4.0/) which permits any noncommercial use, distribution, and reproduction in any medium, provided the original author(s) and the source are credited.
} 
stimulation. Children, 4-6 years of age, with a positive CF newborn screening diagnosis were recruited to complete the $\beta$-adrenergic or cholinergic protocols. CF and CRMS diagnoses were defined by the primary pulmonologist according to current $\mathrm{CF}$ Foundation guidelines. ${ }^{7,9} \mathrm{CF}$ diagnosis required (1) at least one sweat chloride value $\geq 60 \mathrm{mmol} / \mathrm{L}$ and/or (2) 2 CF-causing mutations based on CFTR2 classification. CRMS diagnosis required (1) at least 2 CFTR mutations identified by screening, in which at least one was not classified as $\mathrm{CF}$ causing; (2) asymptomatic health status; and (3) a sweat chloride of $<60 \mathrm{mmol} / \mathrm{L}$ after 6 months of age.

\section{Protocols}

Drugs were prepared on the day of testing and used within $3 \mathrm{~h}$ of preparation to ensure pharmacological activity.

Adult protocol. The dosing of the $\beta$-adrenergic cocktail was the same as the pediatric protocol (below). As described previously, ${ }^{4}$ the same puncture site and lateral needle track were used for sequential subepidermal injections of (1) carbachol $0.01 \mu \mathrm{g}$, (2) atropine $4.4 \mu \mathrm{g}$, and (3) the $\beta$-adrenergic cocktail. Concurrently, atropine was injected into an adjacent site on the same arm where a separate probe recorded the background transpiration rate. Subsequently, the probes were positioned on the other forearm to monitor the peak sweat rate after a single injection of $\beta$-cocktail drugs as well as the background rate (pediatric protocol).
Pediatric protocol. Vital signs were obtained before, immediately after, and $30 \mathrm{~min}$ after testing. In addition, a 3-lead electrocardiogram was used during testing. After coating an area of the volar forearm with mineral oil, an evaporimeter probe (cyberDERM, inc.) was placed over the oiled area to continuously record the unstimulated sweat rate as transepidermal water loss $\left(\mathrm{g} \mathrm{H}_{2} \mathrm{O} / \mathrm{m}^{2} / \mathrm{h}\right)$ (Fig. 1). A second probe was placed on an adjacent oil-coated site and after attaining a stable baseline signal for at least $2 \mathrm{~min}$, the site was cleaned with alcohol and injected as above with a 30-G needle inserted parallel to the skin surface to form a small intradermal wheal with $0.2 \mathrm{~mL}$ of $\beta$-adrenergic cocktail containing the following: $4.4 \mu \mathrm{g}$ atropine (West-Ward, Cheryl Hill, NJ), $450 \mu \mathrm{g}$ aminophylline (Hospira, San Jose, CA), and $2 \mu \mathrm{g}$ isoproterenol (Valeant, Bridgewater, NJ). After the injection, the oil was reapplied and the probe repositioned over the injected area taking care to ensure that needle puncture site was excluded from the area assayed by the probe. Alternatively, in other subjects, only $0.1 \mathrm{~mL}$ of $0.01 \mu \mathrm{g}$ carbachol (Alcon, Forth Worth, TX) was injected. The pediatric doses of drugs in the $\beta$ adrenergic cocktail were half those used previously in adults. ${ }^{4}$ The peak sweat rate was taken as the maximal plateau of sweat rate maintained for at least 2 min preceding a continual decline in rate. Net responses were calculated as the peak response minus the baseline sweat rate. Children watched entertainment videos to help minimize movement. Parents evaluated their child's tolerance of the protocol with a Likert Scale questionnaire. The Children's Hospital Los Angeles Institutional

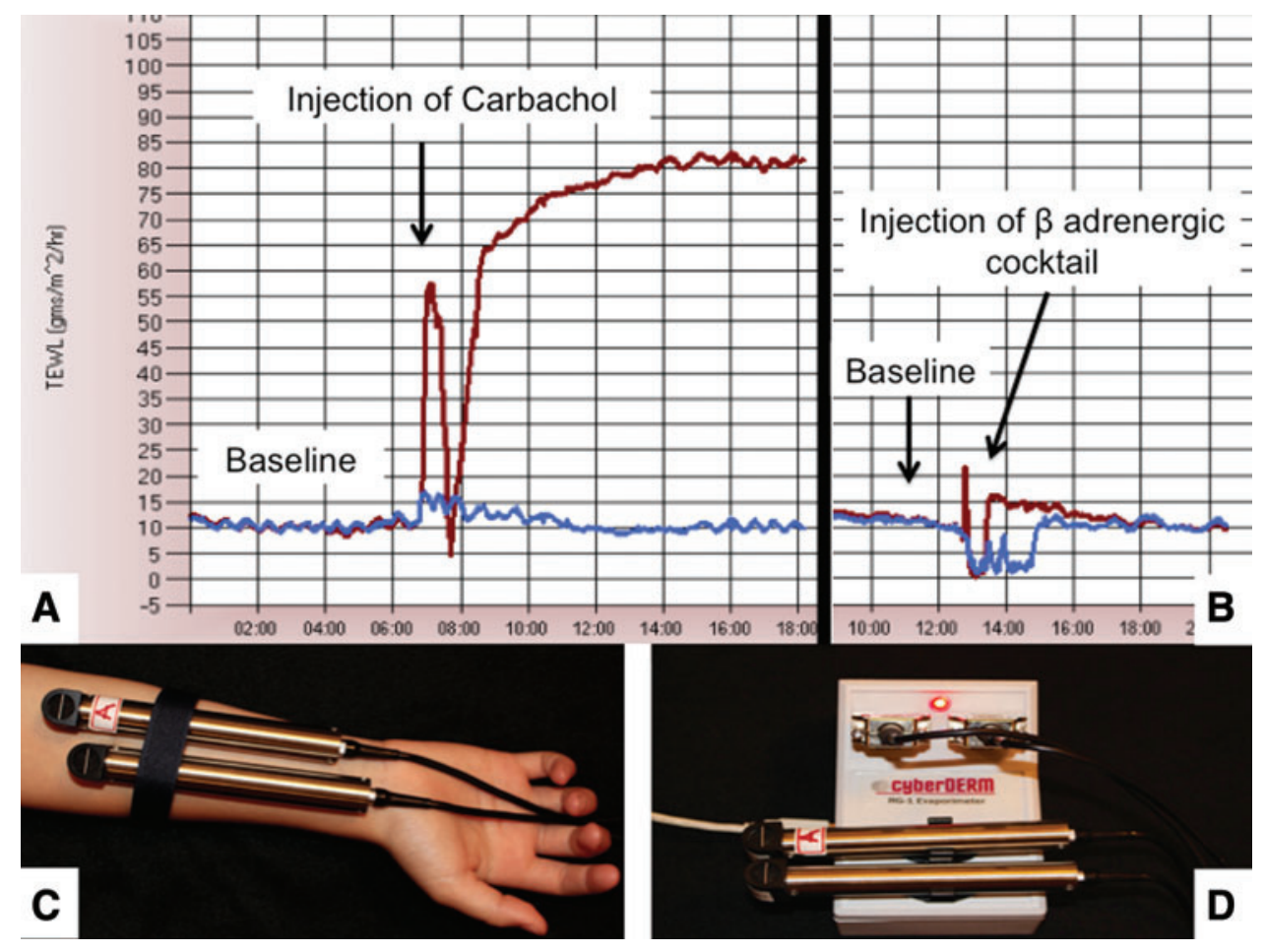

FIG. 1. Example of an evaporimeter measurement in a 4-year-old subject. (A) Water loss was measured as $\mathrm{gm} / \mathrm{m}^{2} / \mathrm{h}$ on the $\mathrm{Y}$-axis over time in minutes on the $\mathrm{X}$-axis. Probe $\mathrm{A}$ and $\mathrm{B}$ were placed as shown in picture $\mathrm{C}$ during testing. Measurement of baseline water loss rate (from 2 to $6 \mathrm{~min}$ ) was followed by abrupt movement artifacts associated with lifting probe A to inject carbachol intradermally. Maximal responses occurred from 14 to 18 min of testing. (B) Trace from same subject on another day. After baseline stabilized at 10-12 min, probe A was lifted to intradermally inject the $\beta$ adrenergic cocktail. Movement artifacts are apparent on both probes. Maximal evaporative $\beta$-response was reported as the mean from 17 to 20 min minus baseline. (C) Probes placed and secured on the volar aspect of the forearm. Systematically, probe A was placed medially and probe B laterally. (D) CyberDerm evaporimeter. Color images available online at www.liebertpub.com/ped 
Review Board (IRB) approved the study. All subjects or parents/legal guardians provided informed consent.

\section{Statistical methods}

Study variables are summarized as mean \pm standard deviation (SD), median, and interquartile range for continuous data, frequency, and percent for categorical data. Comparisons of net peak responses between CF and CRMS groups were made with the Wilcoxon rank sum test. Changes in vital signs were analyzed with repeated measures mixed models.

\section{Results}

A total of 7 healthy adult volunteers and 26 children $(\mathrm{CF}$, $n=16$; CRMS, $n=10$ ) completed the study. Six children were included in both the adrenergic and cholinergic protocol, while 13 children were exclusively included in the adrenergic protocol and 7 were exclusively included in the cholinergic protocol (total of 32 tests in 26 children). Table 1 describes demographic characteristics. Heart rate, respiratory rate, oxygen hemoglobin saturation, systolic/ diastolic blood pressure, and axillary temperature were between the 5th and the 99th percentile for subject's age and gender (Fig. 2). Three-lead electrocardiogram during all 32 tests showed sinus rhythm. Twenty-four of 26 parents (92\%) agreed or strongly agreed that their child tolerated the test well. The parents of the 2 who did not tolerate well justified their answers on the child's anxiety related to the injection.

Mean peak $\beta$-adrenergic responses were similar between adult and pediatric protocols in healthy adult volunteers (adult protocol: $46 \pm 22 \mathrm{~g} \mathrm{H}_{2} \mathrm{O} / \mathrm{m}^{2} / \mathrm{h}$ (mean $\pm \mathrm{SD}$ ) and pediatric protocol: $\left.42 \pm 12 \mathrm{~g} \mathrm{H}_{2} \mathrm{O} / \mathrm{m}^{2} / \mathrm{h}, n=7, p=0.68\right)$. In children, the mean baseline, peak cholinergic, and peak $\beta$-adrenergic between ages of 4 to 6 years were not significantly different between CF and CRMS subjects and were found to be as follows: (1) baseline- $\mathrm{CF}=10.4 \pm 2.9(n=20)$, CRMS $=10.7 \pm 1.9 \quad(n=12 ; p=0.97$, units as above $)$, (2) cholinergic- $\mathrm{CF}=60.3 \pm 23.8 \quad(n=8), \quad \mathrm{CRMS}=57.7 \pm 13.9$ $(n=5 ; p=0.72)$, and (3) $\beta$-adrenergic- $\mathrm{CF}=1.1 \pm 1.7(n=12)$, CRMS $=2.0 \pm 2.0(n=7 ; p=0.14)($ Fig. 3$)$.

\section{Discussion}

This study is a first attempt to assay $\beta$-adrenergic sweating in preschool age children. We found that a modi-

Table 1. Description of the Study Population

\begin{tabular}{|c|c|c|}
\hline Population characteristics & $\begin{array}{c}C F \\
(\mathrm{n}=16)\end{array}$ & $\begin{array}{c}C R M S \\
(\mathrm{n}=10)\end{array}$ \\
\hline $\begin{array}{l}\text { Age (in years) at days of } \\
\text { testing, mean } \pm S D\end{array}$ & $5 \pm 0.8$ & $5 \pm 0.7$ \\
\hline Female gender, $n(\%)$ & $9(56)$ & $5(50)$ \\
\hline $\begin{array}{l}\text { Highest sweat chloride } \\
\quad \text { (in } \mathrm{mmol} / \mathrm{L}), \text { mean } \pm \mathrm{SD}\end{array}$ & $88 \pm 20$ & $26 \pm 14$ \\
\hline $\begin{array}{l}\text { Pancreatic insufficiency, } \\
n(\%)\end{array}$ & $11(69)$ & $0(0)$ \\
\hline $\begin{array}{l}\text { Fecal elastase, median } \\
\quad(\text { IQR), } \mu \mathrm{g} / \mathrm{g}\end{array}$ & $\begin{array}{c}126 \\
(14 \text { to }>500)\end{array}$ & $\begin{array}{l}>500 \\
(491 \text { to }>500)\end{array}$ \\
\hline
\end{tabular}

$\mathrm{CF}$, children with cystic fibrosis; CRMS, children with cystic fibrosis transmembrane conductance regulator-related metabolic syndrome; IQR, interquartile range; $\mathrm{SD}$, standard deviation.

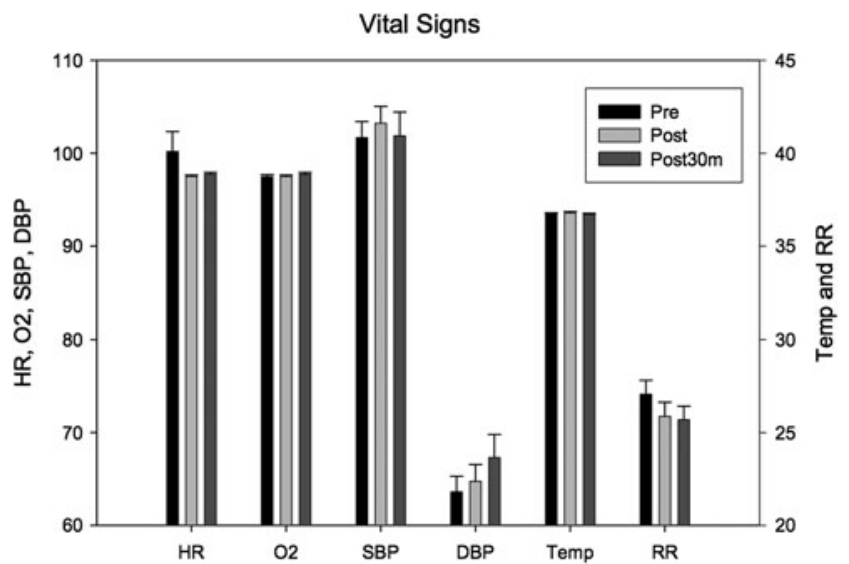

FIG. 2. Vital signs were obtained immediately before and after sweat rate measurements and after $30 \mathrm{~min}$ of observation. Values are mean with standard error of mean (32 tests). DBP, diastolic blood pressure; $\mathrm{HR}$, heart rate; $\mathrm{O}_{2}$, oxygen tension; RR, respiratory rate; SBP, systolic blood pressure; Temp, body temperature.

fied single-injection protocol to stimulate sweating assayed by evaporimetery was safe and well tolerated, but at this stage of development did not discriminate between CF and CRMS subjects. These findings suggest that (1) the minimal detection limit of the evaporimetery-based $\beta$-adrenergic assay is not low enough to detect residual CFTR-dependent sweat activity in CF and CRMS subjects or (2) CFTR protein is also dysfunctional in $\beta$ sweating in CRMS children. Of note, all peak $\beta$ responses in CRMS children were below the cutoff rate $\left(4.5 \mathrm{~g} \mathrm{H}_{2} \mathrm{O} / \mathrm{m}^{2} / \mathrm{h}\right)$ observed in $\mathrm{CF}$ adults, presumed to express little or no CFTR function. ${ }^{4}$ Little is known about CFTR mutations identified in the CRMS population. Among our cohorts, screen-positive subjects had one CF-causing mutation identified as one of the 40 most prevalent CFTR mutations in California (California panel)

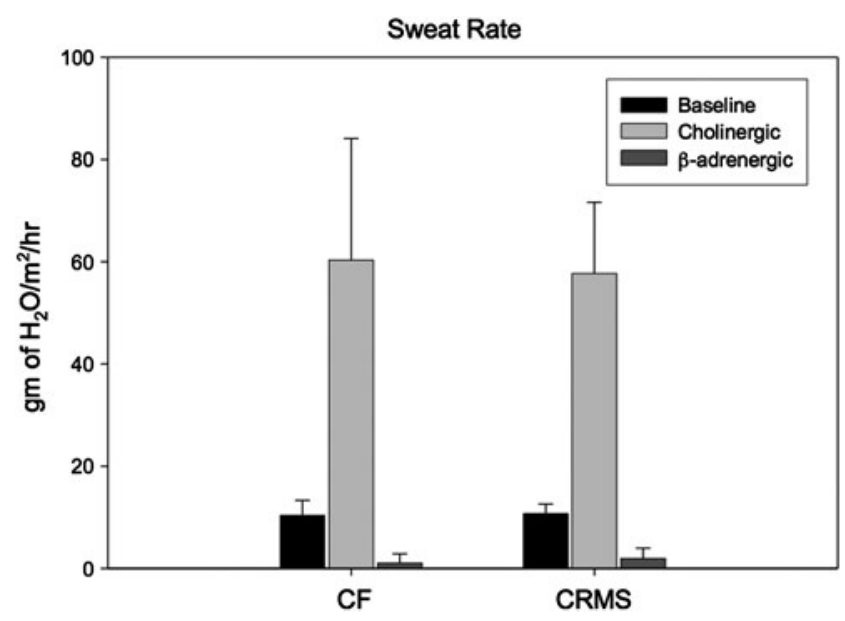

FIG. 3. Sweat rate measurements in response to cholinergic and $\beta$-adrenergic stimulation in the CF and CRMS population (mean \pm standard deviation): baseline, cholinergic, and $\beta$-adrenergic values are reported as deltas (maximal response minus baseline values). CF, cystic fibrosis; CRMS, cystic fibrosis transmembrane conductance regulator-related metabolic syndrome. 
along with a second mutation identified by CFTR - DNA Sanger sequencing (Stanford Molecular Pathology Laboratory). ${ }^{10}$ CRMS subjects had sequenced mutations of unknown clinical significance: L320V, 4375-20A $>$ G, R170H, and c.-510G $>\mathrm{A}$; and others categorized by CFTR2 (www.CFTR2 .org) as "mutations of varying clinical consequence": D1152H, (TG)12-5T, and (TG)11-5T. However, the 5T allele is known to be present in 10-20\% of the general population and results in only $\sim 10 \%$ normal expression of fulllength functional CFTR. ${ }^{11}$ Since most subjects carrying one CF-causing mutation and a $5 \mathrm{~T}$ allele, specifically (TG) 11 and 12, present with a normal phenotype or with CFTRrelated disorder such as combined bilateral absence of vas deferens, ${ }^{12-14}$ we expected $\beta$-adrenergic responses above the cutoff for CF for the 2 subjects with (TG)12-5T. Instead, responses were only 2.95 and $0.18 \mathrm{~g} \mathrm{H}_{2} \mathrm{O} / \mathrm{m}^{2} / \mathrm{h}$, classifying them as CF. Moreover, previous evaporimetry in adults differentiated noncarrier, healthy normal subjects from those with any 2 CFTR mutations, but it did not differenciate CFTR function among those with 2 mutations; that is, CF with pancreatic insufficiency, CF with pancreatic sufficiency, and CFTR-related disorder patients who differ phenotypically; as they all fell below the $4.5 \mathrm{~g} \mathrm{H}_{2} \mathrm{O} / \mathrm{m}^{2} / \mathrm{h}$ cutoff. $^{4}$ Thus, this assay of $\beta$-adrenergic sweat rate should differentiate CRMS children from healthy non-CF children. However, the IRB did not approve participation of healthy controls in this study, which consequently leaves a crucial question of the age at which the $\beta$-adrenergic sweating response develops in children. Lacking evidence to the contrary, we assume that the development of adrenergic and cholinergic pathways is coincident. ${ }^{15}$

A ratiometric assay comparing $\beta$-adrenergic to cholinergic sweat rates using evaporimetry was introduced recently ${ }^{4}$ and a ratiometric optical assay has been used to measure the effect of ivacaftor on single, individual sweat glands in a small sample of subjects carrying G551D and $\mathrm{R} 117 \mathrm{H}{ }^{5,6}$ The $\beta$ response was seen in $16-21 \%$ of sweat glands of medicated patients and in no glands in patients not in therapy, indicating that the single-gland method is a more sensitive measure of sweat response and therefore more cabable of detecting residual CFTR function. ${ }^{6} \mathrm{Un}$ fortunately, monitoring the sweat secretion from individual glands requires complete subject cooperation to accomodate relatively long periods of inactivity and is therefore probably not feasible in young children or infants.

Although most parents agreed this test using a single intradermal injection was tolerable, investigators perceived drug injections as a major limitation in test of young children. A few children presented with significant stress to a single injection, making multi-injection protocols essentially unfeasible.

Since we were limited to a single injection per pediatric subject, we could not "potentiate" the $\beta$-adrenergic response by precholinergic stimulation of the sweat glands as reported for single glands. ${ }^{5}$ However, we found no significant difference in the peak $\beta$-adrenergic responses with or without precholinergic stimulation in adults. Applying only half the dose concentration with or without precholinergic stimulation still stimulated the same range of $\beta$-adrenergic responses reported earlier in 53 healthy controls after cholinergic stimulation $\left(35-50 \mathrm{~g} \mathrm{H}_{2} \mathrm{O} / \mathrm{m}^{2} / \mathrm{h}\right),{ }^{4}$ suggesting that precholinergic stimulation may be unnecessary to prime $\beta$-adrenergic sweat rate responses and, therefore, a single- injection protocol to assess sweat rates in younger children may be acceptable. Another limitation was the small sample size. Considering the consistent null response to $\beta$-adrenergic stimulation in all CRMS children, it was deemed unethical to extend testing with this protocol. Despite the results, $\beta$-adrenergic sweat secretion assays complement the standard quantitative pilocarpine iontophoresis test by uniquely providing a measure of CFTR secretory rather than measuring a strictly absorptive function. ${ }^{5}$

\section{Conclusion}

The $\beta$-adrenergic sweat secretion assay appears safe and feasible for preschool age children. Further technological improvements in sweat detection and minimally invasive drug delivery will likely be required to render test results to the diagnosis and prognosis of CRMS children.

\section{Acknowledgments}

We are thankful to all adults, parents, and children who volunteered for this study. We are grateful for the training received from Julie Avolio, $\mathrm{RN}$-clinical research nurse coordinator and Tanja Gonska, MD-assistant professor at the Division of Gastroenterology, Hepatology, and Nutrition at University of Toronto, Hospital for Sick Children, Toronto, Canada. We also thank A.K.M. Shamsuddin, PhDassistant project scientist, Department of Pediatrics, Division of Respiratory Medicine, University of California, San Diego, for technical assistance and guidance.

Funding: This study received support from the National Institute of Health: SC-CTSI (NIH/NCRR/NCATS; Grant \# KL2TR000131), SC-CTSI Pilot Grant 8UL1TR000130, and the Division of Pediatric Pulmonology at the Children's Hospital Los Angeles, University of Southern California, Keck School of Medicine.

\section{Author Disclosure Statement}

No competing financial interests exist.

\section{References}

1. Reddy MM, Quinton PM. Electrophysiologically distinct cell types in human sweat gland secretory coil. Am J Physiol 1992; 262:C287-C292.

2. Sato K, Sato F. Defective beta adrenergic response of cystic fibrosis sweat glands in vivo and in vitro. J Clin Invest 1984; 73:1763-1771.

3. Behm JK, Hagiwara G, Lewiston NJ, Quinton PM, Wine JJ. Hyposecretion of beta-adrenergically induced sweating in cystic fibrosis heterozygotes. Pediatr Res 1987;22:271-276.

4. Quinton P, et al. beta-adrenergic sweat secretion as a diagnostic test for cystic fibrosis. Am J Respir Crit Care Med 2012;186:732-739.

5. Wine JJ, et al. In vivo readout of CFTR function: ratiometric measurement of CFTR-dependent secretion by individual, identifiable human sweat glands. PLoS One 2013;8:e77114.

6. Char JE, et al. A little CFTR goes a long way: CFTRdependent sweat secretion from G551D and R117H-5T cystic fibrosis subjects taking ivacaftor. PLoS One 2014;9:e88564.

7. Borowitz D, et al. Cystic Fibrosis Foundation practice guidelines for the management of infants with cystic fibrosis transmembrane conductance regulator-related metabolic 
syndrome during the first two years of life and beyond. J Pediatr 2009;155:S106-S116.

8. Ooi CY, et al. Inconclusive diagnosis of cystic fibrosis after newborn screening. Pediatrics 2015;135:e1377-e1385.

9. Borowitz D, et al. Cystic Fibrosis Foundation evidencebased guidelines for management of infants with cystic fibrosis. J Pediatr 2009;155:S73-S93.

10. Kharrazi M, et al. Newborn screening for cystic fibrosis in California. Pediatrics 2015; 136:1062-1072.

11. Chu CS, Trapnell BC, Curristin S, Cutting GR, Crystal RG. Genetic basis of variable exon 9 skipping in cystic fibrosis transmembrane conductance regulator mRNA. Nat Genet 1993;3:151-156.

12. Kiesewetter $\mathrm{S}$, et al. A mutation in CFTR produces different phenotypes depending on chromosomal background. Nat Genet 1993;5:274-278.

13. Noone PG, et al. Lung disease associated with the IVS8 5T allele of the CFTR gene. Am J Respir Crit Care Med 2000;162:1919-1924.

14. Hefferon TW, Groman JD, Yurk CE, Cutting GR. A variable dinucleotide repeat in the CFTR gene contributes to phenotype diversity by forming RNA secondary structures that alter splicing. Proc Natl Acad Sci U S A 2004;101: 3504-3509.

15. Gibson LE. The effect of adrenergic stimulation upon sweating in normal children and cystic fibrosis patients. Pediatrics 1968;42:458-464.

Address correspondence to:

Danieli Barino Salinas, MD, MS

Department of Pediatrics-Pediatric Pulmonology

Children's Hospital Los Angeles

Keck School of Medicine-University

of Southern California (USC)

4650 Sunset Boulevard, MS 83.

Los Angeles, CA 90027

E-mail: dsalinas@chla.usc.edu

Received for publication April 23, 2016; accepted after revision August 4, 2016. 